\title{
Interference between Resonant and Nonresonant Inelastic X-Ray Scattering
}

\author{
Y.-P. Sun, ${ }^{1,2, *}$ Q. Miao, ${ }^{2,3, \dagger}$ A. Pietzsch, ${ }^{4}$ F. Hennies, ${ }^{5,6}$ T. Schmitt, ${ }^{7}$ V. N. Strocov, ${ }^{7}$ J. Andersson, ${ }^{6,8}$ B. Kennedy, ${ }^{5}$ \\ J. Schlappa, ${ }^{4}$ A. Föhlisch, ${ }^{4,9}$ F. Gel'mukhanov, ${ }^{2}$ and J.-E. Rubensson ${ }^{6}$ \\ ${ }^{1}$ School of Science, Shandong University of Technology, ZiBo, 255049 Shandong, People's Republic of China \\ ${ }^{2}$ Theoretical Chemistry, School of Biotechnology, Royal Institute of Technology, S-106 91 Stockholm, Sweden \\ ${ }^{3}$ College of Physics and Electronics, Shandong Normal University, 250014 Jinan, People's Republic of China \\ ${ }^{4}$ Institute for Methods and Instrumentation in Synchrotron Radiation Research G-ISRR, \\ Helmholtz-Zentrum Berlin für Materialien und Energie, Albert-Einstein-Straße 15, 12489 Berlin, Germany \\ ${ }^{5}$ MAX IV Laboratory, Lund University, Box 118, 22100 Lund, Sweden \\ ${ }^{6}$ Department of Physics and Astronomy, Uppsala University, Box 516, S-751 20 Uppsala, Sweden \\ ${ }^{7}$ Swiss Light Source, Paul Scherrer Institut, CH-5232 Villigen PSI, Switzerland \\ ${ }^{8}$ Centre for Quantum Technologies, National University of Singapore, 3 Science Drive 2, 117543 Singapore, Singapore \\ ${ }^{9}$ Institut für Physik und Astronomie, Universität Potsdam, Karl-Liebknecht-Strasse 24-25, 14476 Potsdam, Germany
}

(Received 22 November 2012; published 29 May 2013)

\begin{abstract}
A detailed study of inelastic x-ray scattering from the ground state to the ${ }^{3} \Sigma_{g}\left(3 \sigma_{g}^{-1} 3 s_{g}^{1}\right)$ state of the $\mathrm{O}_{2}$ molecule is presented. The observed angular anisotropy shows that the vibrational excitations within this final state are strongly dependent on the polarization of the incident radiation. The analysis demonstrates that this is a manifestation of interference between resonant and direct nonresonant inelastic x-ray scattering. This interference provides a new tool to monitor nuclear dynamics by relative rotation of the polarization vectors of the incident and scattered photons.
\end{abstract}

DOI: 10.1103/PhysRevLett.110.223001

The nonresonant scattering channel has so far been neglected when considering resonant inelastic scattering (RIXS) in the sub-keV range. On the other hand, the direct nonresonant (Thomson) scattering channel significantly contributes to resonant elastic scattering [1-5]. Nonresonant inelastic x-ray scattering (NIXS) is prominent in the hard $\mathrm{X}$-ray range, and is exploited in well-established materials science methods [6-9]. To the best of our knowledge, however, a demonstration of the influence of the nonresonant channel on resonant inelastic scattering phenomena is lacking also for hard $\mathrm{x}$ rays.

Here we show that NIXS influences scattering deep in the soft X-ray range, in the spectrum of the $\mathrm{O}_{2}$ molecule excited at the $1 s \rightarrow \sigma^{*}$ resonance [10-12]. NIXS interference with the RIXS channel is reflected in the polarization dependence of vibrational excitations within a welldefined electronic final state. This phenomenology is an indication of NIXS-RIXS interference because the NIXS and RIXS channels have different polarization dependence and induce different nuclear dynamics. The observed angular anisotropy is pronounced, demonstrating that NIXSRIXS interference is important in this case and suggesting that NIXS should be considered when analyzing state-ofthe-art resonant $\mathrm{X}$-ray scattering spectra in general.

The observed polarization dependence of the vibrational excitations is a consequence of NIXS-RIXS interference and does not indicate that the Born-Oppenheimer approximation is violated. In spite of the formal analogy with the interference between the resonant channel and the channel of nonresonant direct ionization in resonant Auger scattering, there is a qualitative difference between the two
PACS numbers: 33.20.Rm, 32.80.Aa, 33.20.Fb, 33.80.Gj

processes. The interference between the resonant channel and the channel of direct ionization is a typical non-BornOppenheimer effect [13-16]. Here the final state wave function cannot be written as a product of electronic and nuclear wave functions due to the mixing between the degenerate autoionizing core-excited state and the continuum state in which it is embedded. The picture is qualitatively different for $\mathrm{x}$-ray Raman scattering where the energy spacing between the final and the core-excited state is very large $(\approx 524 \mathrm{eV})$. In the absence of external fields, the nuclear motion in the gerade final state is independent of the nuclear dynamics in the ungerade core-excited state. However, the final and core-excited states are brought together by the two-color x-ray field: The final state, directly populated via the NIXS channel, mixes with the core-excited state through the RIXS channel which ends up in the same final state, and thus the nuclear dynamics of these states becomes entangled. Here we demonstrate that NIXS-RIXS interference seen in the polarization dependence of the vibrational profile can be monitored by varying the propagation direction of the scattered photon relative to the polarization vector of the incident photon.

The experiment was performed at the ADRESS beam line [17] of the Swiss Light Source, Paul Scherrer Institut Villigen, using the SAXES spectrometer [18]. The overall energy resolution was around $50 \mathrm{meV}$, allowing for the separation of individual vibrations $[4,11,19,20]$. Linearly polarized incident radiation with the polarization vector $\mathbf{e}$ parallel and perpendicular to the scattering plane was used: $\theta=\angle\left(\mathbf{e}, \mathbf{k}_{1}\right)=0^{\circ}$ and $\theta=90^{\circ}$, where $\mathbf{k}_{1}$ is the momentum of the scattered photon. The spectrometer measured 
scattered photons with all polarization states, $\mathbf{e}_{1}$, and the angle between the momenta of scattered and incident $\mathrm{x}$-ray photons was $\chi=\angle\left(\mathbf{k}_{1}, \mathbf{k}\right)=90^{\circ}$, corresponding to a momentum transfer of $q \approx 2 k \sin (\chi / 2)=\sqrt{2} k\left(\mathbf{q}=\mathbf{k}_{1}-\mathbf{k}\right)$. Gas-phase measurements were facilitated using a flow cell with a $100 \mathrm{~nm}$ thick diamondlike window, separating the ultrahigh vacuum from the sample gas. Incoming and outgoing radiation passed through the same window, both at an angle of $45 \mathrm{deg}$.

The $X^{3} \Sigma_{g}^{-}$ground state of $\mathrm{O}_{2}$ has the electronic configuration $1 \sigma_{g}^{2} 1 \sigma_{u}^{2} \cdots 3 \sigma_{g}^{2} 1 \pi_{u}^{4} 1 \pi_{g}^{2}$. At excitation energy $\hbar \omega=539 \mathrm{eV}$ the crossing of the potential surfaces of the dissociative $i={ }^{3} \Sigma_{u}^{-}\left(1 \sigma_{g}^{-1} 3 \sigma_{u}^{1}\right)$ and the bound $r=$ ${ }^{3} \Sigma_{u}^{-}\left(1 \sigma_{u}^{-1} 3 s_{g}^{1}\right)$ core-excited states is reached [21-23]. The resulting scattering spectra, shown on the $\Delta \hbar \omega=\hbar \omega_{1}-$ $\hbar \omega$ energy loss scale in Fig. 1, comprise scattering to vibrational excitations in the $X^{3} \Sigma_{g}^{-}$electronic ground state $(-12 \mathrm{eV} \leq \Delta \hbar \omega \leq 0)$, to the repulsive ${ }^{3} \Pi_{g}\left(1 \pi_{u}^{-1} 3 \sigma_{u}^{1}\right)$ state $(\Delta \hbar \omega \approx-14 \mathrm{eV})$ and to the ${ }^{3} \Sigma_{g}^{-}\left(3 \sigma_{g}^{-1} 3 s_{g}^{1}\right)$ state $(\Delta \hbar \omega \approx-14.9 \mathrm{eV})$. The narrow resonance near $\Delta \hbar \omega \approx$ $-12.2 \mathrm{eV}$ is the so-called atomic peak $[3,24]$ related to the decay of the core-excited isolated oxygen atom following dissociation [11] (Fig. 2). Before discussing the scattering to the $f={ }^{3} \Sigma_{g}^{-}\left(3 \sigma_{g}^{-1} 3 s_{g}^{1}\right)$ final state in detail we first comment on the theoretical method and the polarization dependence in RIXS.

Potential energy surfaces of the ground and dissociative core-excited states (Fig. 2) were computed using complete active space wave-function based approaches. The potential energy surface of the core-excited state was determined using the CASSCF-MRPT2 method, in which all 16 electrons of $\mathrm{O}_{2}$ are included in an active space consisting of 10 orbitals used in the wave-function optimization, and dynamic electron correlation effects were accounted for using multireference second-order perturbation theory (MRPT2). The potential curve of the ${ }^{3} \Sigma_{g}^{-}\left(3 \sigma_{g}^{-1} 3 s_{g}^{1}\right)$ final

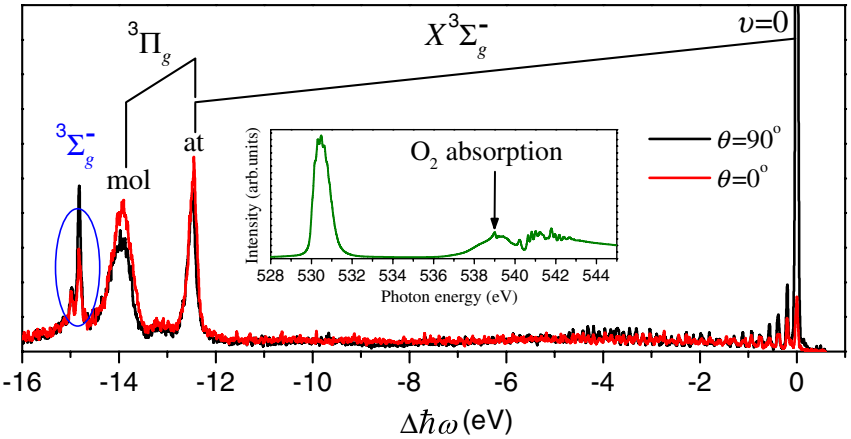

FIG. 1 (color online). High-resolution experimental RIXS spectrum of molecular oxygen excited at the dissociative $1 s \rightarrow$ $\sigma^{*} 3 \Sigma_{u}$ resonance $(\hbar \omega=539 \mathrm{eV})$ measured in two geometries, $\theta=\angle\left(\mathbf{e}, \mathbf{k}_{1}\right)=0^{\circ}$ and $90^{\circ}$. The scattering to the $X^{3} \Sigma_{g}^{-}$and ${ }^{3} \Pi_{g}$ final states has opposite polarization dependence. Due to ultrafast dissociation a narrow atomic (at) peak appears in addition to the molecular (mol) peaks. state was approximated by a harmonic potential with the experimental vibrational frequency $0.15 \mathrm{eV}$ and the same equilibrium distance (2.42 a.u.) as the ionic $3 \sigma_{g}^{-1}$ state [25]. These values are close to what is predicted by our $a b$ initio calculations ( $0.147 \mathrm{eV}$ and 2.45 a.u.). For the bound core-excited ${ }^{3} \Sigma_{u}^{-}\left(1 \sigma_{u}^{-1} 3 s_{g}^{1}\right)$ Rydberg state, we have used the potentials from Ref. [23].

The scattering anisotropy in RIXS is very sensitive to the symmetry of the involved molecular orbitals [3]. As seen in Fig. 1, the RIXS cross section $\sigma_{R}$ for scattering to the final states of $\Sigma$ and $\Pi$ symmetry shows opposite $\theta$ dependence. This is in agreement with the theoretical prediction $[11,26]$ that $\sigma_{R}(\Sigma) \propto 1+\sin ^{2} \theta$ and $\sigma_{R}(\Pi) \propto 4-\sin ^{2} \theta$. The elastic peak ( $\Delta \hbar \omega=0)$ which corresponds to the scattering to the fundamental vibrational state $\nu=0$ of the electronic ground state shows strong and qualitatively different anisotropy than the scattering to vibrationally excited states. The reason for this is the interference between the RIXS and strong Thomson scattering channels which affects only the elastic resonance $(\nu=0)[2-5]$.

In the following we focus on the scattering to the ${ }^{3} \Sigma_{g}^{-}\left(3 \sigma_{g}^{-1} 3 s_{g}^{1}\right)$ state (Fig. 3). In accordance with our simulations, we assign the two narrow peaks around $\Delta \hbar \omega \approx$ $-14.9 \mathrm{eV}$ to the vibrational levels $\nu=0$ and $\nu=1$ of this final state.

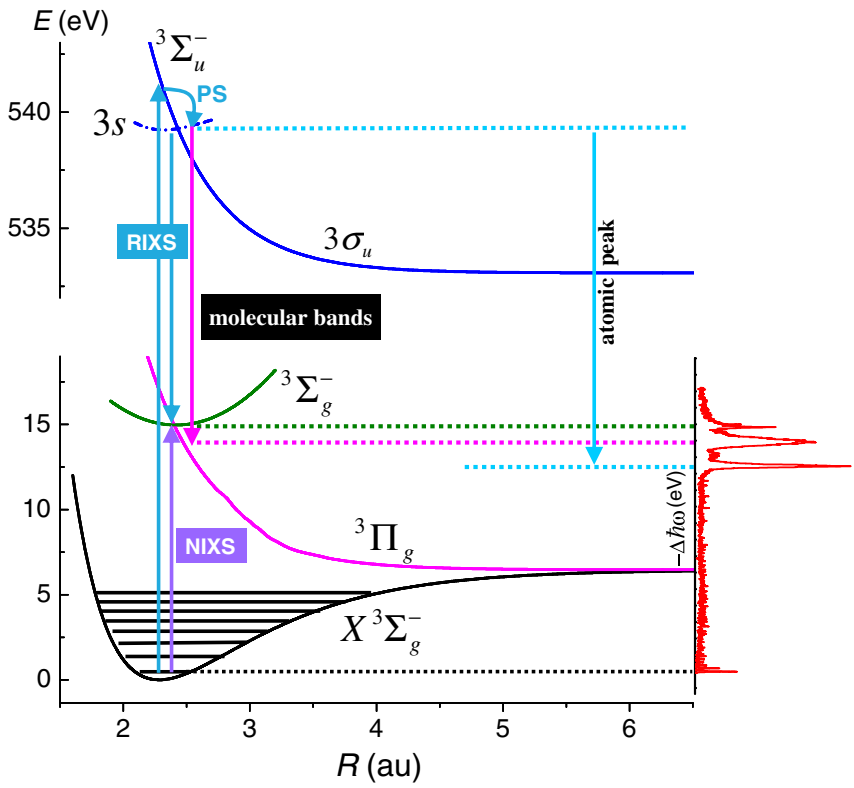

FIG. 2 (color online). Scheme of transitions responsible for the formation of the RIXS spectrum shown in Fig. 1. The excitation energy is set to reach the potential curve crossing of the dissociative state and a bound Rydberg core-excited state. The scattering to the electronic ground state $X^{3} \Sigma_{g}^{-}$forms the extensive vibrational profile in the region $-12 \mathrm{eV} \lesssim \Delta \hbar \omega<0$ and ends up by a narrow atomic peak. The molecular band at $\Delta \hbar \omega \approx$ $-14 \mathrm{eV}$ is due to the scattering to the ${ }^{3} \Pi_{g}$ final state. The corevalence parity swap (PS) $\left|1 \sigma_{g}^{-1} 3 \sigma_{u}^{1}\right\rangle \rightarrow\left|1 \sigma_{u}^{-1} 3 s_{g}^{1}\right\rangle$ opens the RIXS channel to the ${ }^{3} \Sigma_{g}\left(3 \sigma_{g}^{-1} 3 s_{g}^{1}\right)^{3} \Sigma_{g}^{-}$final state. 


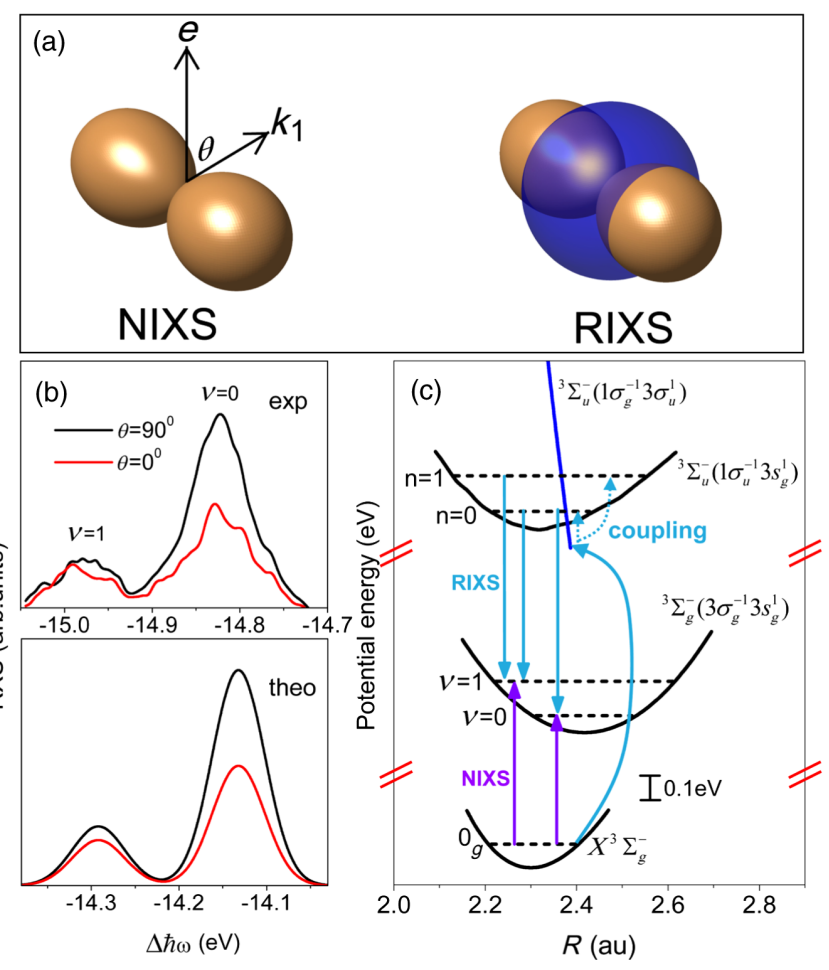

FIG. 3 (color online). The upper panel (a) shows the scattering anisotropy of the NIXS $\left(\sin ^{2} \theta\right)$ and RIXS $\left(1+\sin ^{2} \theta\right)$ channels. (b) The observed polarization dependence of $x$-ray scattering to the $\nu=0$ and $\nu=1$ vibrational levels of the ${ }^{3} \Sigma_{g}^{-}\left(3 \sigma_{g}^{-1} 3 s_{g}^{1}\right)$ final state is in agreement with the theoretical prediction, when interference between the RIXS and NIXS channels is taken into account. (c) A detailed view of the potential curves of the states involved in the interfering RIXS and NIXS $\left(3 \sigma_{g} \rightarrow 3 s\right)$ channels. RIXS comprises three steps: $1 \sigma_{g} \rightarrow 3 \sigma_{u},\left|1 \sigma_{g}^{-1} 3 \sigma_{u}^{1}\right\rangle \rightarrow$ $\left|1 \sigma_{u}^{-1} 3 s^{1}\right\rangle$, and $3 \sigma_{g} \rightarrow 1 \sigma_{u}$.

The amplitudes for the NIXS and RIXS channels are given $[1,3]$ by the first and second term of the interaction Hamiltonian, $V=\alpha^{2}\left(\mathbf{A} \cdot \mathbf{A}_{1}\right)-\alpha\left[\mathbf{p} \cdot\left(\mathbf{A}+\mathbf{A}_{1}\right)\right]$, respectively. Here $\mathbf{A}$ and $\mathbf{A}_{1}$ are the vector potentials of the incoming and scattered photons, $\mathbf{p}$ is the momentum operator of electrons, and $\alpha=1 / 137$. The two contributions have different scattering anisotropy [Fig. 3(a)] and form the total scattering amplitude:

$$
\mathcal{F}_{\nu}=\left(\mathbf{e}_{1} \cdot \mathbf{e}\right) F_{N, \nu}+\left(\mathbf{e}_{1} \cdot \mathbf{n}\right)(\mathbf{e} \cdot \mathbf{n}) F_{R, \nu}
$$

The polarization anisotropy in RIXS depends on the orientation of $\mathbf{e}$ and $\mathbf{e}_{1}$ relative to the dipole moments of the core excitation and decay which are parallel to the unit vector along the molecular axis, $\mathbf{n}$. The NIXS transition from the $3 \sigma_{g}$ to the $3 s_{g}$ orbital has the amplitude $F_{N, \nu}=$ $\left\langle f\left|\sum e^{\imath \mathbf{q} \cdot \mathbf{r}}\right| g\right\rangle\langle\nu \mid 0\rangle \approx\left\langle 3 \sigma_{g}\left|e^{\imath \mathbf{q} \cdot \mathbf{r}}\right| 3 s_{g}\right\rangle\langle\nu \mid 0\rangle$, where $\mathbf{q}$ is the momentum transfer. It is dipole forbidden, and we take nondipole effects into account by making a Taylor expansion of $e^{\imath \mathbf{q} \cdot \mathbf{r}}$ up to second order:

$$
\begin{aligned}
F_{N, \nu} \approx & -\left[\alpha_{2 p} 2 \sin \left(\frac{\mathbf{q} \cdot \mathbf{R}}{2}\right)\left\langle 2 p_{z}|\mathbf{q} \cdot \mathbf{r}| 3 s\right\rangle\right. \\
& \left.+\alpha_{2 s} q^{2}\left\langle 3 s\left|r^{2}\right| 2 s\right\rangle\right]\langle\nu \mid 0\rangle \\
\approx & -\left[(\hat{\mathbf{q}} \cdot \mathbf{n})^{2} F_{N, \nu}^{a}+F_{N, \nu}^{\mathrm{is}}\right] .
\end{aligned}
$$

The NIXS amplitude is explicitly written in terms of its anisotropic and isotropic contributions, $F_{N, \nu}^{a}=$ $\alpha_{2 p} q^{2} R d_{2 p_{z}, 3 s}\langle\nu \mid 0\rangle$ and $F_{N, \nu}^{\text {is }}=\alpha_{2 s} q^{2}\left\langle 3 s\left|r^{2}\right| 2 s\right\rangle\langle\nu \mid 0\rangle$, respectively. Here $\alpha_{i}=C_{i}^{\left(3 \sigma_{g}\right)} C_{3 s}^{\left(3 s_{g}\right)}(i=2 s, 2 p), \hat{\mathbf{q}}=\mathbf{q} / q$, and $\mathbf{R}=R \mathbf{n}$ is the internuclear radius vector, $C_{m}^{\left(3 \sigma_{g}\right)}$ and $C_{m}^{\left(3 s_{g}\right)}$ are the amplitudes of the atomic wave function $(m=$ $\left.2 s, 2 p_{z}, 3 s\right)$ in the $3 \sigma_{g}$ molecular and $3 s_{g}$ Rydberg orbitals, respectively, and $\langle\nu \mid 0\rangle$ is the Franck-Condon amplitude between the vibrational levels of the final and ground states. As seen in Eq. (2), the $3 \sigma_{g} \rightarrow 3 s_{g}$ NIXS amplitude is due to the $2 s \rightarrow 3 s$ atomic quadrupole transition and the two-center interference given by the $\sin (\mathbf{q} \cdot \mathbf{R} / 2)$ factor. With this interference term, the dipole approximation is violated when the $\mathrm{x}$-ray wavelength approaches the size of the molecule, $q R \sim 1(q R / 2 \sim k R \sim 0.3$ in our case $)$. This simulates the nondipole behavior of RIXS in the hard $\mathrm{x}$-ray region [3,26-28].

One reason that the NIXS channel becomes important in this specific case is that the RIXS amplitude $F_{R, \nu}$ is small, as seen in the experimental data (Fig. 1). Indeed, the ratio of the intensity of the discussed transition to the total intensity of the atomic and molecular bands is about $10^{-1}$.

As we have shown earlier [11], the population of the $f={ }^{3} \Sigma_{g}\left(3 \sigma_{g}^{-1} 3 s_{g}^{1}\right)$ final state via the $1 \sigma_{g} \rightarrow 3 \sigma_{u}$ resonance requires a hole-electron parity swap in the core-excited state [11]. This is accomplished by the interaction $\beta \equiv$ $\left\langle r\left|r_{12}^{-1}\right| i\right\rangle \sim 0.1 \mathrm{eV}$ between the initially excited $i=$ $1 \sigma_{g}^{-1} 3 \sigma_{u}^{1}$ dissociative state and the $r=1 \sigma_{u}^{-1} 3 s_{g}^{1}$ Rydberg state [14,21-23] [Fig. 3(c)]. The resonant propagator $K_{n, \epsilon}^{r i}=\beta\langle n \mid \epsilon\rangle /\left[\omega-\left(E_{r, n}-E_{g, 0}\right)+\imath \Gamma\right]$ of a hole-electron parity swap modifies [11] the conventional expression of the RIXS amplitude [3]:

$$
F_{R, \nu}=\omega_{1} \omega \int d \epsilon \frac{d_{f r}\langle\nu \mid n\rangle K_{n, \epsilon}^{r i}\langle\epsilon \mid 0\rangle d_{i 0}}{\omega-\left(E_{i, \epsilon}-E_{g, 0}\right)+{ }_{\imath} \Gamma} .
$$

The Franck-Condon amplitudes $\langle\epsilon \mid 0\rangle,\langle n \mid \epsilon\rangle$, and $\langle\nu \mid n\rangle$ describe the transitions from the ground state to the dissociative state $|\epsilon\rangle$, from the dissociative to the $n$th vibrational level in the bound Rydberg state, and from this level to the vibrational level $|\nu\rangle$ of the final state. Here, $d_{i 0}$ and $d_{f r}$ are the transition dipole moments of the core excitation and radiative decay, respectively, $E_{i, \epsilon}$ is the energy of the intermediate dissociative state, and $\hbar \Gamma=0.07 \mathrm{eV}$ is the lifetime broadening of the core-excited state.

The total scattering cross section summed over polarizations of the final photon and averaged over molecular orientations is 


$$
\sigma_{\nu}=\frac{r_{0}^{2} \omega_{1}}{15 \omega}\left|F_{R, \nu}\right|^{2}\left(1+\rho_{\nu} \sin ^{2} \theta\right) \Phi .
$$

Here, $r_{0}$ is the classical radius of the electron, $\rho_{\nu}=$ $\left[5 / 4 \Delta_{N, \nu}^{2}+\Delta_{N R, \nu}^{2}+\left|F_{R, \nu}^{\prime \prime}\right|^{2}\right] /\left|F_{R, \nu}\right|^{2}, F_{R, \nu}=F_{R, \nu}^{\prime}+\imath F_{R, \nu}^{\prime \prime}$, $\Delta_{N, \nu}=F_{N, \nu}^{a}-F_{N, \nu}^{\text {is }}$, and $\Delta_{N R, \nu}=F_{R, \nu}^{\prime}-1 / 2\left(F_{N, \nu}^{a}+5 F_{N, \nu}^{\text {is }}\right)$, and $\Phi=\Phi\left(\Delta \omega+\omega_{f, \nu ; 0,0}\right)$ is the experimental spectral function which includes both monochromator and spectrometer contributions. The dependence on the angle $\theta=$ $\angle\left(\mathbf{e}, \mathbf{k}_{1}\right)$ is qualitatively different for the NIXS channel $\sigma_{N} \propto \sin ^{2} \theta$ [Fig. 3(a)] which vanishes when $\theta=0$, contrary to the RIXS channel [11,26] $\sigma_{R} \propto 1+\sin ^{2} \theta$. This, together with the fact that the vibrational profiles are also very different for NIXS $(\langle\nu \mid 0\rangle)$ and RIXS $\left(\langle\nu \mid n\rangle K_{n, \epsilon}^{j i}\langle\epsilon \mid 0\rangle\right)$ channels, explains why the vibrational profile strongly depends on $\theta$ [Fig. 3(b)]. This dependence is given by the anisotropy parameter $\rho_{\nu}$, which, according to simulations, is very sensitive to the vibrational level of the final electronic state: $\rho_{0}=0.8$ and $\rho_{1}=0.3$. One should notice that the vibrational profile ceases to depend on the polarization when the NIXS is absent $\left(F_{N, \nu}=0\right)$ because the anisotropy parameter becomes the same $\rho_{\nu}=1$ for all vibrational levels.

In conclusion, we have shown that vibrationally resolved scattering anisotropy in the inelastic $\mathrm{x}$-ray scattering spectra of the oxygen molecule reveals prominent interference between the resonant and nonresonant scattering channels. RIXS-NIXS interference is possible also at higher photon energies, and based on simulations we estimate that it can be observed, e.g., in x-ray Raman scattering at the $\mathrm{Cl} K$ edge of the $\mathrm{HCl}$ molecule.

This work was performed at the ADRESS beam line using the SAXES instrument jointly built by the Paul Scherrer Institut, Switzerland and Politecnico di Milano, Italy. The research leading to these results has received funding from the European Community's Seventh Framework Programme (FP7/2007-2013) under Grant Agreement No. 226716 and from the Helmholtz Virtual Institute "Dynamic Pathways in Multidimensional Landscapes." The support by the Swedish Research Council (VR), Carl Tryggers Stiftelse (CTS), National Natural Science Foundation of China (Grants No. 11204163 and No. 10974121), and National Basic Research Program of China (2011CB808100) is gratefully acknowledged.

*sunyuping821008@163.com

†qmiao@theochem.kth.se

[1] Resonant Anomalous X-Ray Scattering: Theory and Applications edited by G. Materlik, C. J. Sparks, and K. Fischer (North-Holland, Amsterdam, 1994).

[2] F. Gel'mukhanov and H. Ågren, Phys. Rev. A 56, 2676 (1997).
[3] F. Gel'mukhanov and H. Ågren, Phys. Rep. 312, 87 (1999).

[4] A. Pietzsch et al., Phys. Rev. Lett. 106, 153004 (2011).

[5] S. Carniato, P. Selles, L. Journel, R. Guillemin, W. C. Stolte, L. El Khoury, T. Marin, F. Gel'mukhanov, D. W. Lindle, and M. Simon, J. Chem. Phys. 137, 094311 (2012).

[6] R. Verbeni, M. D’Astuto, M. Krisch, M. Lorenzen, A. Mermet, G. Monaco, H. Requardt, and F. Sette, Rev. Sci. Instrum. 79, 083902 (2008).

[7] K. Hämäläinen and S. Manninen, J. Phys. Condens. Matter 13, 7539 (2001).

[8] M.W. Haverkort, A. Tanaka, L. H. Tjeng, and G. A. Sawatzky, Phys. Rev. Lett. 99, 257401 (2007).

[9] D. Sokaras et al., Rev. Sci. Instrum. 83, 043112 (2012).

[10] P. Glans, K. Gunnelin, P. Skytt, J.-H. Guo, N. Wassdahl, J. Nordgren, H. Ågren, F. Gel'mukhanov, T. Warwick, and E. Rotenberg, Phys. Rev. Lett. 76, 2448 (1996).

[11] Y.-P. Sun et al., J. Phys. B 44, 161002 (2011).

[12] V. Kimberg, T. Gejo, M. Oura, T. Tokushima, Y. Horikawa, H. Arai, S. Shin, and N. Kosugi, Phys. Rev. A 85, 032503 (2012).

[13] J. L. Dehmer, P. M. Dehmer, J. B. West, M. A. Hayes, M. R. F. Siggel, and A.C. Parr, J. Chem. Phys. 97, 7911 (1992).

[14] I. Hjelte et al., J. Chem. Phys. 122, 084306 (2005).

[15] C. Miron, V. Kimberg, P. Morin, C. Nicolas, N. Kosugi, S. Gavrilyuk, and F. Gel'mukhanov, Phys. Rev. Lett. 105, 093002 (2010).

[16] Ph. V. Demekhin, I. D. Petrov, V.L. Sukhorukov, W. Kielich, A. Knie, H. Schmoranzer, and A. Ehresmann, Phys. Rev. Lett. 104, 243001 (2010).

[17] V. N. Strocov et al., J. Synchrotron Radiat. 17, 631 (2010).

[18] G. Ghiringhelli et al., Rev. Sci. Instrum. 77, 113108 (2006).

[19] F. Hennies, A. Pietzsch, M. Berglund, A. Föhlisch, T. Schmitt, V. Strocov, H. O. Karlsson, J. Andersson, and J.-E. Rubensson, Phys. Rev. Lett. 104, 193002 (2010).

[20] Y.-P. Sun et al., Phys. Rev. B 84, 132202 (2011).

[21] J. Adachi, N. Kosugi, and A. Yagishita, J. Phys. B 38, R127 (2005).

[22] R. Feifel et al., J. Chem. Phys. 128, 064304 (2008).

[23] Y. Velkov, V. Kimberg, N. Kosugi, P. Sałek, and F. Gel'mukhanov, Chem. Phys. Lett. 476, 147 (2009).

[24] P. Morin and C. Miron, J. Electron Spectrosc. Relat. Phenom. 185, 259 (2012).

[25] K.P. Huber and G. Herzberg, Molecular Spectra and Molecular Structure IV (Van Nostrand Reinhold, New York, 1979).

[26] F. Gel'mukhanov and H. Ågren, Phys. Rev. A 49, 4378 (1994).

[27] J. D. Mills, J. Sheehy, T. Ferrett, S. Southworth, R. Mayer, D. Lindle, and P. Langhoff, Phys. Rev. Lett. 79, 383 (1997).

[28] F. Gel'mukhanov and H. Ågren, Phys. Rev. Lett. 82, 666 (1999). 